the history of the patient's previous illnesses are valuable aids. With German measles smallıpox need never be confused, but in any difficulty the above indications are sufficient. The rash of scarlatina differs very widely in its appearance and circumstances from variola.

The Erdption in Mird Modiried Casts.

$r$ The eruption of chicken-pox may be distinguished from small-pox by the absence of premonitory fever, the rash being the first, of ten the only, symptom noticed in the former disease. Moreover, the papular stage is very transient, giving rise in a few hours (as compared with two clear days) to a clear vesicle on a slightly inflamed base, without induration. The face is not, as in variola, the most favourite place, and different stages of the eruption may always in chicken-pox be seen at the same time.

It should always be borne in mind that small-pox as we see it in the present day presents many degrees of severity, and some cases are so trivial as scarcely to merit the patient's notice, from the scanty rash and few symptoms, much less to impede him in his work. These cases require to be diagnosed from acne. This can only be done (a) by the position, for acne favours the roots of the hair, and $(b)$ by the fact that the acne spots may generally be seen in several stages, and some nearly always show the small points of comedones. In the mildest case of small-pox some constitutional signs may generally be discovered by careful investigation. A few isolated papules occurring (and not becoming vesicular or pustular) on the fourth or fifth day of an illness are most probably small-pox.

\section{Agr OF THE RASH.}

I should like to take this opportunity of pointing out the great value which attaches to the question of time in the diagnosis of a small-pox rash. A knowledge of the exact age of an eruption will often decide the question. At the end of twelve hours the papules of measles begin to fade, those of small-pox get harder and larger, and after an interval of two days become vesicular; whereas varicella is vesicular almost at the very commencement.

\section{Characters on Palpation.}

The feel of a small-pox rash is another point only second in importance to the element of time. To pass your hand over the forehead and cheeks is a procedure which should never be omitted in a doubtful case. In this way the disease could almost be diagnosed in the dark. I know objections may be urged, but the advantages are very great, for the hard shotty lumps of small-pox, which can be felt even before they. are visible, are totally different from the feel of the soft flat marginated papules of measles, or the non-indurated vesicles of varicella.

These are the chief diseases which in actual practice are liable to be confused with incipient small-pox. At a later stage there is rarely much difficulty, though $I$ have mistaken a case of acute glanders, in which the usual nasal discharge was absent, and my mistake was amply confirmed. But having regard to the grave issues at stake, it is undoubtedly wise to adopt quarantine precautions in any doubtful case. It is sincerely to be hoped, moreover, that the day is not far distant when a properly fitted quarantine ward will be regarded as a necessary adjunct to the receiving ward of every isolation hospital.

\section{CASE OF ACUTE PERIOSTITIS OF THE JAW FROM MERCURIAL POISONING.}

BY W. H. C. STAVELEY, F.R.C.S.ENG., L.R.C.P.LoNd. $\triangle N D$

R. DENISON PEDLEY, L.D.S., F.R.C.S.EDIN.

Madical Report by Mr. Staveury.

Mrs. K., widow, aged 31, had had fairly good general health until recently. She had had a good deal of worry from legal matters in connection with her late husband's afairs. She had suffered occasionally from muscular rheumatism.
She consulted me for the first time on December 23rd, 1892, complaining of general nervousness, tremors of the hands, inability to sleep, and almost complete want of appetite. She attributed her condition to two boils which had formed on the right arm. One of these was well, the other had been incised and was healing. She also had some facial neuralgia. The catamenia appeared regularly every three weeks. The bowels acted twice daily. She was a tall, spare woman. There was marked pallor of the face, but not much anæmia of the mucous membranes. The tongue was thickly coated and the breath offensive. Teeth had been recently filled, and she was in the habit of visiting her dentist every six months.

I attributed her condition to insufficient food, and prescribed a generous diet and the following mixture: Ferr. et quin. cit. gr. $x_{\text {., }}$ tinct. nuc. vom. $m x$, aq. chloroform. $z_{j}$, t. d. s.

On December 26 th she sent for me, as the neuralgia had been very severe. The breath was very offensive, the gums swollen, and there was much creamy fur about the teeth. I advised her to visit her dentist if the pain did not subside. and prescribed pulv. phenacetin, gr. 5 , to be repeated in half an hour's time if necessary.

On December 27 th I received a note from Mr. R. Denison Pedley at 8 P.M., saying that the patient had been to see him and that he had extracted two teeth to relieve pain. He suggested that the condition of the mouth was due to some poison, probably septic. I saw Mrs. $K$. the same evening, and found the teeth on the right side of the: mouth all loose, pus welling up from the sockets; the breath was most offensive, and there was a profuse flow of saliva. The temperature was $100^{\circ}$.

On December 28 th she was somewhat relieved; the temperature was $99^{\circ}$. A chlorine mouth wash was ordered to be used frequently.

On January 2nd, 1893, there was still much discharge, and great pain in the second (?) lower molar; pus was still welling up from the tooth sockets. At 5 P.M. I met Mr. Pedley in consultation. She was then free from pain, and we decided to wait. We discussed the question of poison for some time, but could discover no source. We were both much perplexed as to the cause. She continued under my treatment until the end of January. Abscesses formed at the roots of two other teeth, with grea's pain, necessitating their removal. All the teeth extracted were carefully examined, and the pulps found to be healthy.

On February 4th the patient's sister informed me for the first time that the patient had been using a lotion for hex complexion since the beginning of October, 1892, and left $a$. bottle of it at my house. On examining it I found it contained mercury, apparently in large quantities. I did not. attempt to estimate it quantitatively. I left this bottle at the office of the British Medical Association on February 14th, 1893.

Dental Report by Mr. Prdify.

The following is the dental report from notes taken at the time or the day after:

December 27th, 1892. Mrs. K., aged about 30, came to-day with her sister about 4 P.M. Complains of constant pain in upper and lower jaw on the right side; has kept her awake at night for nearly a week. Says she hardly knows how to bear the pain ; it is acute and throbbing. On examination patient can scarcely open her mouth. The breath is very offensive. From the mid line in upper and lower jaws on right side, as far as the wisdom teeth, the gums are inflamed and thick. ened. The teeth are all loose, and on pressing the gum pus oozes out round the necks of the teeth. The molar teeth in upper and lower jaw on right side are acutely sensitive when pressed upon. Patient fainted twice during the examination. Decided to remove a molar tooth in upper and lower $j a w$ on right side, the most sensitive. Nitrous oxide administered. Upper twelve years' molar and lower wisdom tooth removed on the right side. Patient seemed much relieved. Teeth not carious. Teeth carefully examined after extraction. The upper molar had been previously stopped on the posterior surface, but there was no reason for supposing that the nerve pulp was irritated. The periosteum was much inflamed and thickened. On cutting the teeth open the pulps were healthy in appearance.

December 29th, 1892. The father of Mrs: K. came to-day, 
and asked if $I$ would visit Mrs. $K$., as she was too ill to come out. Went at 3 P.M. Mouth in same condition as before, but much relieved by extraction. Jooks very ill, and seems very weak.

January 23rd, 1893. Mrs. K. came this afternoon. Inflammation of upper and lower jaw seems to have spread to the left side. The teeth are sensitive and loose. A lower bicuspid tooth on left side, acutely sensitive, and, although not carious, the patient complains of constant pain. Nitrous oxide administered; tooth extracted. Periosteum very much thickened and deeply congested. On cutting it open, the nerve pulp was apparently quite healthy.

January 25th, 1893. Mrs. K. came this afternoon. Pus still oozing out round necks of teeth ; there is a slight improvement; breath still offensive. Right lower molar acutely sensitive and very loose; extracted under gas. Periosteum inflamed and thickened; nerve pulp seems quite healthy. Patient complains of great pain, but can open her mouth a little wider.

February 17th, 1893. Mrs. K. came to-day. Mouth healthy ; no pain; breath sweet; teeth much tighter; took models of upper and lower jaw.

REMARKs.-Mrs. $\dot{K}$. has periodically consulted me with regard to her teeth during the past two years. I have always regarded her as a healthy woman with a healthy mouth. The symptoms point to a general inflammation of the jaws, in which the teeth were secondarily affected. The teeth were extracted as the readiest means of relieving tension, and by so doing the patient has been probably saved from necrosis of the jaws. The sudden onset, the condition of the mouth, gams, and teeth, the fotor of breath, the whole history, in fact, points clearly to poisoning by mercury, and that I believe it to be. During the past fourteen years I have seen many cases both in hospital and private practice, but in all my experience I have never seen a patient suffer such intolerable and persistent pain.

The following is the patient's statement: "During the early part of October, 1892, I went to Mme. Anna Ruppert, in Regent Street, my complexion not being good; otherwise I was in good health. She looked at my face with a magnifying glass, and said that her preparation would remove spots. She rec smmended me to try her special treatment. Next morning she sent me by parcel post three bottles of lotion, three cakes of spap, one pot of ointment, one box of powder, for which I paid two guineas. The interview lasted about five minutes. About a month after I had been to her I began to be sleepless, lost my appetite, my hands became so tremulous that several of my friends noticed it. I was formerly much constipated, but the bowels now acted about twice a day. Two boils formed on my right arm about the beginning of December. I rubbed some of the ointment on a scratch on my arm; boils immediately followed. On December 23rd I went to consult Mr. Staveley. Except for being run down and in bad health, he did not know what was the matter with me. I complained to him of what I thought was neuralgia in the right side of my face and head; this got worse and worse, and I suffered agonies in my mouth. Inflammation set up, and on December 27 th I went to Mr. Pedley. He extracted one tooth from the upper jaw, and one from the lower; he could not account for the state of my mouth, but thought I had been poisoned. On two occasions I fainted; once when I went to Mr. Pedley. All my teeth were loose for some time, and the pain was intense. The flow of saliva was so bad that I could not swallow it. I was in my room for four or five weeks, and getting better, but had another attack of violent pain. I had another tooth out on January 23rd, 1893, ; on the 25 th or 26th the fourth and last was extracted. I may add that there was constant discharge from the gums, and I was unable to eat solid food for several weeks."

Remarks BY Mr. Staveler.-I am of opinion that the case was one of mercurial poisoning, and that it was only by relieving tension by removing the teeth that the patient escaped necrosis of the jaw. I have failed to discover any other way that the patient could have absorbed mercury except by the lotion. I feel convinced that the lotion was the cause of the illness.

\section{ME M:ORA N DA: MEDICAL, SURGICAL, OBSTETRICAL, THERA- PEUTICAL, PATHOLOGICAL, Etc.}

\section{A CASE OF TRANSPOSITION OF THE VISCERA.}

M. A. B., a female, aged 14 years, is fairly well developed, being $4 \mathrm{ft} .4$ in. in height, and 6 st. 2 lbs. in weight. I have known her for nine years, and have attended her for an ulcer of the cornea, and epidemic influenza, the only ailments she has had during that time. Previous to this she had no illness of any moment. The patient seems to suffer no inconvenience from her abnormal state, and has no dyspnoea even on exertion.

The Heart.-On inspection a wavy impulse is seen in the second, third, and fourth interspaces on the right side. The apex beat is in the fifth right intercostal space, 1 in. below the nipple line, and 13 in. from mid-sternum. On palpation the area of the apex beat is normal. On percussion the superficial cardiac dulness begins at the fourth rib on the right side, the deep at the upper border of the third rib. The transverse dulness begins $\frac{1}{3}$ in. to the right of the sternum, and extends across for $3 \frac{1}{4}$ in. On auscultation there is no pericardial murmur. At the apex is heard a high-pitched systolic murmur, which is conducted a short way into the axilla and very distinctly andible in the back, at a point internal to and above the angle of the right scapula. A loud systolic murmur is heard in the second right interspace. In the third interspace, at a point $1 \frac{1}{2}$ in. from the margin of the sternum, a slight roughness follows the second sound.

The Liver is on the left side. Hepatic dulness begins at the sixth intercostal space, and extends down for $3 \frac{1}{4}$ in. In the mid-axillary line it extends from the eighth rib. Hepatic dulness is absent on the right side.

The spleen is on the right side; otherwise normal. The urine is normal. The patient seems to be the only member of the family with transposed viscera.

Shepshed, Leicestershire.

G. Cardno Still, M.B.

\section{REPORTS}

ox

MEDICAL \& SURGICAL PRACTICE IN THE HOSPITALS AND ASYLUMS OF GREAT BRITAIN, IRELAND, AND THE COLONIES.

SAMARITAN FREE HOSPITAL, LONDON.

PREGNANCY COMPLICATED BP TWO OVARIAN TUMOURS : MISCARRIAGE : SUBSFQUENT REMOVAL OF TWO GANGRENOUS DERMOIDS : RECOVERY.

(By A. C. Butler-Smythe, F.R.C.S.Edin., Surgeon to Outpatients ; and Surgeon to the Grosvenor Hospital for Women and Children.)

Is May, 1892, Dr. Ensor, of North Kensington, was called in to see a woman, aged 30 , who had suddenly been taken ill with excruciating pains in her abdomen, and who was supposed to be miscarrying. He examined her, and finding "an abdominal swelling and an unusual pelvic condition" sent her to the Samaritan Hospital, where she was admitted, a bed having been kindly placed at my disposal by my senior colleague, Dr. Bantock. The patient was a slender lighthaired woman of medium height, who appeared to have gone through much suffering, her body, limbs, and face being much emaciated.

History. - She had been married twelve years, and was the mother of two children, besides which she had become pregnant three times within three years, but in each pregnancy had aborted about the third month, the last occasion being two and a-half years ago. Her second infant died soon after its birth, and she was in bed for over a month with inflammation of both breasts, followed by "peritonitis." Ever since that time her health has been bad, and for two years or more she had been losing flesh. In October, 1891, an attack of rheumatic fever kept her in bed for five weeks. For 